\title{
Mathematical modeling of aerodynamic processes in railway tunnels on high-speed railways
}

\author{
Alexander Ledyaev ${ }^{1}$, Vladimir Kavkazskiy ${ }^{1}$, Yan Vatulin ${ }^{1}$, Valeriy Svitin ${ }^{1}$, and Oleg \\ Shelgunov ${ }^{1 *}$ \\ ${ }^{1}$ Emperor Alexander I Petersburg State Transport University, Moskovsky ave, 9, Saint Petersburg, \\ 190031, Russia
}

\begin{abstract}
The study of aerodynamic processes in railway tunnels on highspeed railways in the absence of practical experience in operation should be carried out with a sufficient degree of accuracy only by mathematical modeling. A multi-factor experiment was performed, which resulted in solving the optimization task of determining the tunnel cross-sectional area, taking into account aerodynamic processes. Based on the analysis of the results, the conclusion is made about the applicability of the method to the prognosis of aerodynamic effects on prospective tunnel structures of high-speed railways and optimization of geometric parameters of the tunnels.
\end{abstract}

\section{Introduction}

Aerodynamic processes in high-speed train traffic make the design of the cross-sectional area of tunnels one of the most difficult issues in tunnel construction. This parameter significantly affects the cost of tunnels, which can vary by $20-30 \%$, respectively, on the feasibility of construction. To determine the factors affecting the geometric parameters of structures, taking into account the aerodynamics, two possible solutions of the problem are used - methods of physical and mathematical modeling.

Physical modeling has been used to solve aerodynamics tasks since the end of the XIX century [1]. There are methods of research in aerodynamic tubes (wind tunnels) based on the principle of movement reversibility and similarity theory - it involves blowing air flow (air injection devices) of a stationary model; as well as "centrifugal" modeling, in which the air flow is generated by the object model, which allows simulating inertial interactions. These methods are reasonably applicable for measuring the values of pressure on the object surfaces, acting forces, studying the influence of temperatures and acoustic fields, and visualizing flows from the point of view of hydromechanics and aeromechanics. However, they have a number of disadvantages, including inappropriate similarity of the generated air flow and similarity criteria for other parameters (flow velocity and models, geometry of research objects), as well as complexity. For these reasons, it is impossible to use methods

\footnotetext{
*Corresponding author: ooshelgunov@gmail.com
} 
for fully solve the tasks of aerodynamic interaction between the train and infrastructure objects. In the absence of experience in designing tunnels on high-speed railways (HSR), mathematical modeling becomes the only way.

Mathematical modeling of aerodynamic processes of interaction of various objects is a relatively new way of solving tasks, used since the 70-80 of the XX century [2,3]. Today, the software allows with sufficient accuracy to predict and to reproduce the aerodynamic processes in the conditions of high speed traffic. Existing methods and their combinations are the main apparatus of researchers who set various tasks, which allows for comparative analysis $[3,4,5,6,9,12]$. However, many studies contain assumptions, inaccuracy and incomplete dependencies between the geometric and mechanical parameters of a tunnel and a high-speed train and the environment, which can lead to insufficient physical similarity of processes. Such inaccuracies in modeling and the lack of domestic analogues of modeling are the reasons for the necessity of a full-factor experiment - the investigation of aerodynamic processes in railway tunnels on the HSR, the interaction of the multi-factor system " tunnel-train».

\section{Description of the research methodology}

The purpose of the study is to substantiate the parameters of the cross-section area of tunnels on the HSR depending on the trains velocity, taking into account aerodynamic processes, as well as to determine whether the geometric parameters of tunnel structures meet the aerodynamic requirements, and to confirm the proposed hypothesis of the behavior of the multi-factor system "tunnel-train" in high-speed traffic.

Objectives of the study: the determination of aerodynamic parameters and loads when the train moves in the tunnel on the HSR in the set-based optimization plan for the range of parameters of multi-factor system "tunnel train" and optimization of the multi-factor system "tunnel-train" at the appointed criterion.

The hypothesis of the internal interaction of the multi-factor system "tunnel-train" in high-speed traffic, put forward on the basis of the aerodynamic theory terms:

- the ability to control aerodynamic effects within the "tunnel-train" system by changing the parameters of the tunnel, taking into account the constancy of the accepted parameters of the train.

The complex of numerical researches is a combination of solutions to external and internal tasks of aerodynamics using the finite volume method. In this study, the solution of the set research tasks of aerodynamic interaction in high-speed train traffic is carried out using the train "reverse" model, which is developed and successfully tested by specialists of the Petersburg State Transport University (PSTU) in 2017. Its essence is as follows: determining the aerodynamic parameters on the surface of the train from the air flow on the principle of a wind tunnel, the load received on the surface of the train model will first be applied to the train itself, thus the train will be endowed with the ability of the object to generate pressure waves in accordance with the similarity principle. The sequential solution of external and internal tasks allows determining the aerodynamic load values on various objects in the zone of influence of a moving train generating pressure amplitude with sufficient accuracy. The advantage of this method is its simplicity and the ability to take into account various properties of transport infrastructure, train body and the environment, which is perfectly correlated with the tasks and the requirement to take into account the factors of aerodynamic interaction in high-speed train traffic, so its applicability to the solution of the set tasks is justified.

Three-dimensional modeling of the multi-factor system "tunnel-train" in high-speed traffic with differentiated parameters is carried out using the SolidWorks complex in the FlowSimulation extension. The complex allows analyzing and prediction the properties and 
behavior of objects in the environment of real liquids and gases. FlowSimulation implements a finite-volume approach for approximating the equations of a mathematical model. To calculate the behavior of air masses in the software environment, a system of Navier-Stokes equations averaged by Reynolds (RANS) is applied to each elementary volume of air for three-dimensional non-stationary flows. Two-parameter $\mathrm{k}-\varepsilon$ models can be assigned as turbulence models, where $\mathrm{k}$ is the relative kinetic energy, and $\varepsilon$ is the velocity of viscous kinetic energy dissipation, which makes it possible to use this device for describing turbulent flows to solve aerodynamic tasks of interaction of the multi-factor tunnel-train system in high-speed traffic.

Before modeling the aerodynamic effects in the tunnel and calculating the results of the aerodynamic effects of the train in the open space were successfully verified, the model was calibrated. Then the multi-factor system "tunnel-train" was simulated in high-speed traffic conditions, taking into account the aerodynamics. The results of calculations are verified with existing foreign normative values, as well as with similar studies $[7,8]$.

\section{Research procedure and discussion of results}

In order to study the aerodynamic interaction of the multi-factor system "tunnel-train", a series of experiments was carried out. The study includes modeling the movement of a high-speed train in tunnel in a virtual sealed environment under various initial conditions: mechanical and geometric parameters of the system - high-speed train velocity $\mathrm{v},(\mathrm{km} / \mathrm{h})$, tunnel cross-sectional area $\mathrm{S}_{\text {tun }},\left(\mathrm{m}^{2}\right)$ and tunnel length $\mathrm{L},(\mathrm{m})$, reduced to a dimensionless length ratio $\lambda$ (the division of the length of the tunnel $\mathrm{L}_{\mathrm{tun}}$ to the length of the train $\mathrm{L}_{\mathrm{tr}}$ ).

The boundary conditions are set to the parameters of the environment within a limited virtual space, namely: temperature $T^{\circ} 293(\mathrm{~K})$, pressure p 101,325 $(\mathrm{Pa})$, density $\rho 1.204$ $\left(\mathrm{kg} / \mathrm{m}^{3}\right)$, kinematic viscosity $\vee 1.5 \cdot 10^{-5}\left(\mathrm{~m}^{2} / \mathrm{s}\right)$, dynamic viscosity $\eta 18.1(\mathrm{mPa} \mathrm{s})$. The $k$ cturbulence models with $\mathrm{k}=1, \varepsilon=1$ values was chosen. High-speed train model - realwall element, roughness $\mu 6.0$ (mcm). Tunnel model -realwall element, roughness $\mu 5.0$ ( $\mathrm{mcm})$.

The research was carried out in accordance with the optimal planning of the experiment program. To implement a complete factor experiment, the D - optimality criterion is used, which allows to investigate the nonlinear dependencies that are associated with the aerodynamic parameters, with a confidence level equals 0.95 . Table 1 shows the experimental conditions specified in certain ranges: the high-speed train velocity within $200-400 \mathrm{~km} / \mathrm{h}$ (the interval is determined by the conditions of high-speed train traffic), the cross-sectional area of the tunnel $-75-110 \mathrm{~m}^{2}$ (the interval is determined by the recommended values in the technical specifications developed including the PSTU (2014)), the length ratio - 1-12 (the interval is determined in accordance with regulatory documents and studies as the most influential on the amount of aerodynamic effects).

Table 1. Initial data of variables for experiments

\begin{tabular}{|c|c|c|c|}
\hline \multirow{3}{*}{$\begin{array}{c}\text { № } \\
\text { experiment }\end{array}$} & \multicolumn{3}{|c|}{ Variablequantity } \\
\hline & $\mathrm{X} 1$ & $\mathrm{X} 2$ & X3 \\
\hline & $\mathrm{V}, \mathrm{km} / \mathrm{h}$ & $\mathrm{S}, \mathrm{m}^{2}$ & $\lambda$ \\
\hline 1 & 400 & 110 & 7 \\
\hline 2 & 400 & 98.5 & 12 \\
\hline 3 & 200 & 86.5 & 3 \\
\hline 4 & 400 & 110 & 1 \\
\hline 5 & 200 & 75 & 12 \\
\hline 6 & 200 & 110 & 1 \\
\hline 7 & 400 & 75 & 12 \\
\hline
\end{tabular}




\begin{tabular}{cccc}
8 & 200 & 98.5 & 7 \\
\hline 9 & 266.5 & 86.5 & 1 \\
\hline 10 & 400 & 75 & 1 \\
\hline 11 & 200 & 110 & 12 \\
\hline 12 & 200 & 75 & 1 \\
\hline 13 & 266.5 & 110 & 3 \\
\hline 14 & 333.5 & 75 & 3 \\
\hline
\end{tabular}

The mathematical model of the multi-factor system "tunnel-train" consist a verified model of a high-speed train and a universal tunnel structure (figure 1). The tunnel structure is modeled as an infrastructure object with a rigid ballastless track, the tunnel structure is a permanent precast lining with a thickness of $350 \mathrm{~mm}$, the internal equipment and arrangement is reproduced in a minimum amount, since the purpose of the study is to analyze the aerodynamic pressure on the surface of the tunnel lining.

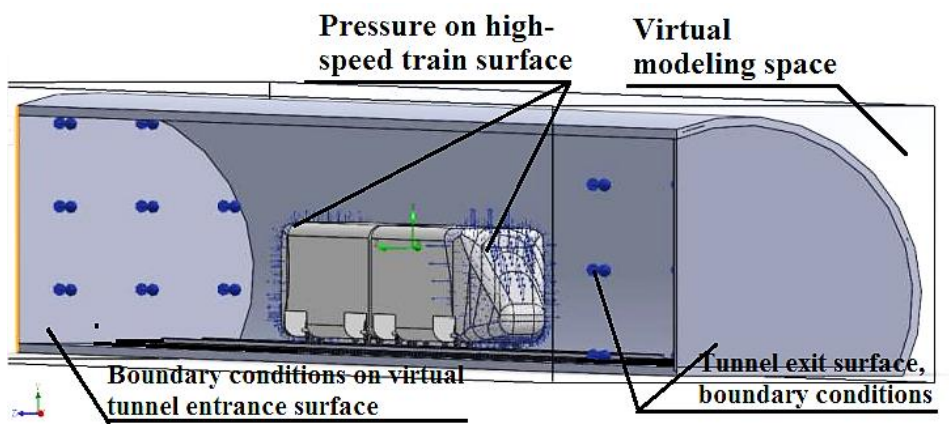

Fig.1. Mathematical model-high-speed train in a tunnel

Analysis of research results includes verification with solutions obtained by other researchers and normative values. To verify the adopted model and its calibration, studies were conducted with initial data corresponding to the initial data for solving certain tasks of aerodynamic interaction of a high-speed train. Initial conditions: train velocity v $=350 \mathrm{~km} /$ $\mathrm{h}$, cross-sectional area $S_{\text {tun }}=75.0 \mathrm{~m}^{2}$, length ratio $\lambda=5$. This approach allows debugging the model of the multi-factor system "tunnel-train" at the initial stages of modeling in order to avoid further errors: they can lead to significant time costs.

Table 2 compares the results of solving aerodynamic interaction tasks [9] with the results of the study (solution 3 corresponds to the method described in this paper). The reliability of the qualitative picture of the studied phenomena and the quantitative convergence within the accepted value of confidence probability is shown, which proves the possibility of fullfledged application of the model for the study of the aerodynamic interaction of the multifactor system "tunnel-train".

Table 2. Comparison of the results obtained in the simulation of the tunnel passage by train in highspeed traffic conditions

\begin{tabular}{llll}
\hline \multicolumn{2}{c}{ Measured parameter } & \multicolumn{3}{c}{ Solution methods } \\
\cline { 2 - 4 } & Solution 1 & Solution 2 & Solution 3 \\
\hline $\begin{array}{l}\text { Static pressure } \\
\text { amplitude, } \mathrm{Pa}\end{array}$ & $2,820 / 100 \%$ & $2,601 /-7.8 \%$ & $2,935 /+4.1 \%$ \\
\hline Maximum pressure, $\mathrm{Pa}$ & $6,710 / 100 \%$ & $6922 /+3.2 \%$ & $6,415 /-4.4 \%$ \\
\hline
\end{tabular}


The internal tasks of aerodynamic interaction of a high-speed train and a tunnel structure were solved in accordance with the experimental planning program. The results of solving the tasks were numerical values and pictures (Fig.2,3,4,5) values such as pressure drop amplitudes, aerodynamic drag, aerodynamic load on the tunnel lining, and air flow characteristics.

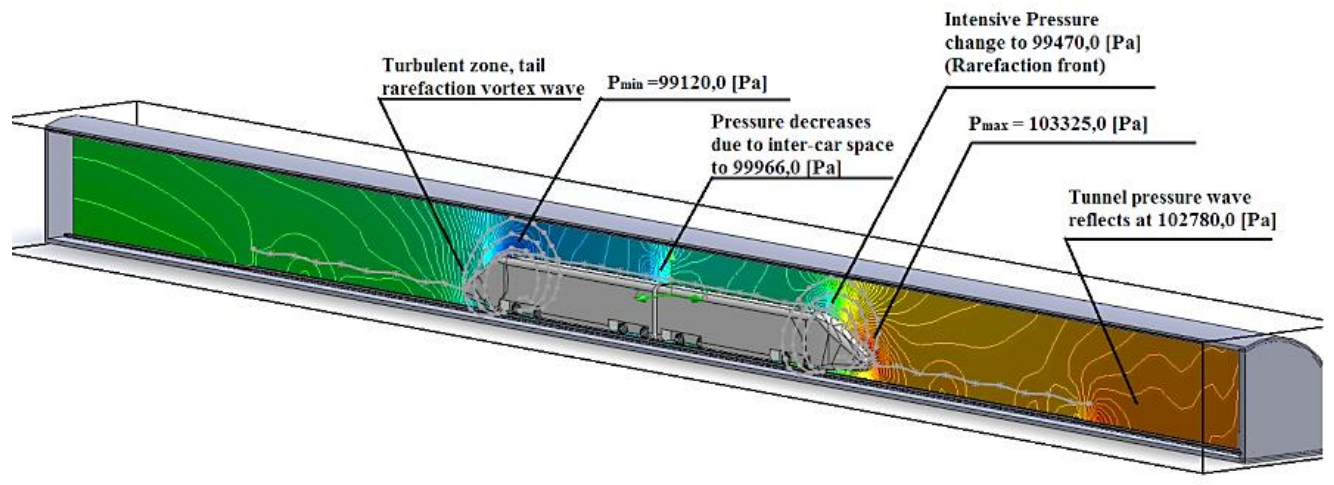

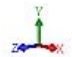

Fig.2. Resulting flow pressure diagram in the tunnel when the train is moving at $333.5 \mathrm{~km} / \mathrm{h}$

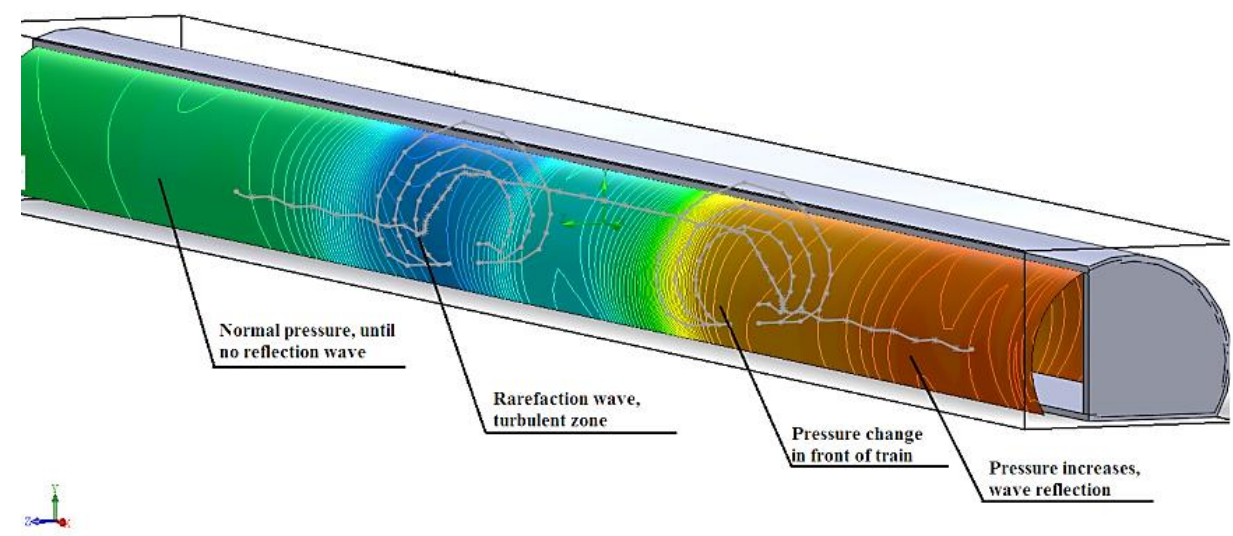

Fig.3. Resulting flow pressure diagram on the tunnel lining when the train is moving at $333.5 \mathrm{~km} / \mathrm{h}$

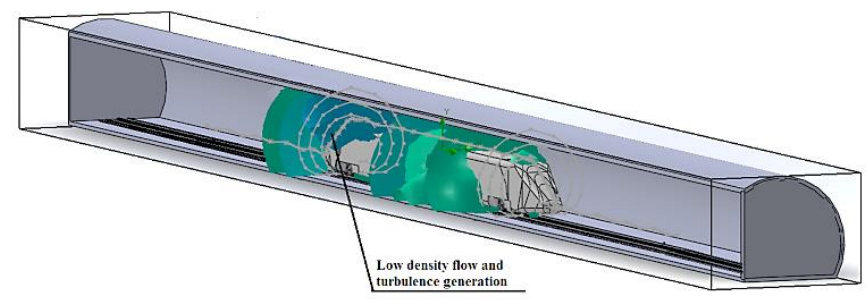

Fig.4. Volumetric diagram of densities of the entrained air flow in the tunnel 


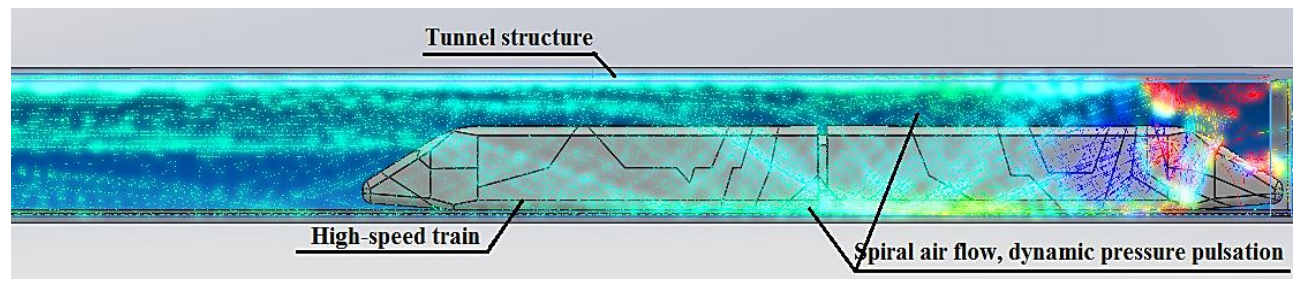

Fig. 5. Vortex of the air flow in a spiral, creating pressure pulsations

It is noted that in determining the physics of the aerodynamic process, well-known observations and assumptions are confirmed. For example, the statements presented by the researchers $[2,3,10,11,12]$ were identified, namely:

- confirmed the phenomenon of reflection of tunnel pressure waves propagating over the entire length of the tunnel during the movement of the train;

- velocity of air flow reaches $135 \%$ of the train velocity;

- significant masses of air are intensively drawn from the compression zone at the head of the train to the rarefaction zone at the tail of the train, forming turbulent flows of lower density (Fig.4);

- the pressure acting on the surface of the tunnel lining is not constant along the length of the tunnel and the passage time of the composition, which is confirmed by pulsations of the air flow, the pressure is dynamic (Fig.5).

Graphical interpretation of the results of the mathematical study contributed to verification with known dependencies [8]. The convergence of the quantitative parameters of physical phenomena was proved: comparison of the values of pressure values showed a satisfactory convergence (7-12\%), which is considered acceptable in the field of aerodynamic research. Thus, the possibility of switching to the task of optimizing the multifactor system "tunnel-train" is determined.

The aim of the optimization task due to the possible redundancy of the tunnel cross sectional area value in high-speed train traffic conditions, which, subject to compliance with all standard requirements to structures should be removed, as the excess crosssectional area of the tunnel increases the cost of construction. The following position is assigned as a criterion for the optimization task: the minimum cross-sectional area of the tunnel at the maximum train velocities in the high-speed interval, taking into account compliance with the safety and aerodynamics criteria (limiting the upper value of the permissible aerodynamic pressure drop). In the given initial conditions, load values are determined for the transition to solving the optimization task and determining the crosssection area of the tunnel, and regression analysis is performed.

Regression analysis of the aerodynamic process within the "tunnel-train" system is based primarily on certain correlations of the theory of aerodynamics, known and proven by researchers $[2,11]$. Then as well as- onthe previously unexplored in the aggregate of the multi-factor system "tunnel-train" assumed dependencies that allow for a complete factor experiment. Establishing full-factor dependencies will allow prediction of the behavior of the "tunnel-train" system when variables vary and apply the results practically to the evaluation of structures.

As characteristic dependencies, graphs of the influence of the mechanical parameters of the system under study on the geometric ones are shown, thus showing the degree of dependencies of the system parameters (Fig.6.7). In figure 6, the graph shows the increase of pressure as an increase in velocity and a decrease in the cross-sectional area of the tunnel. The value of $\mathrm{R}^{2}$ corresponds to the value of the approximation accuracy, thus indicating sufficient accuracy of the constructed dependencies. 


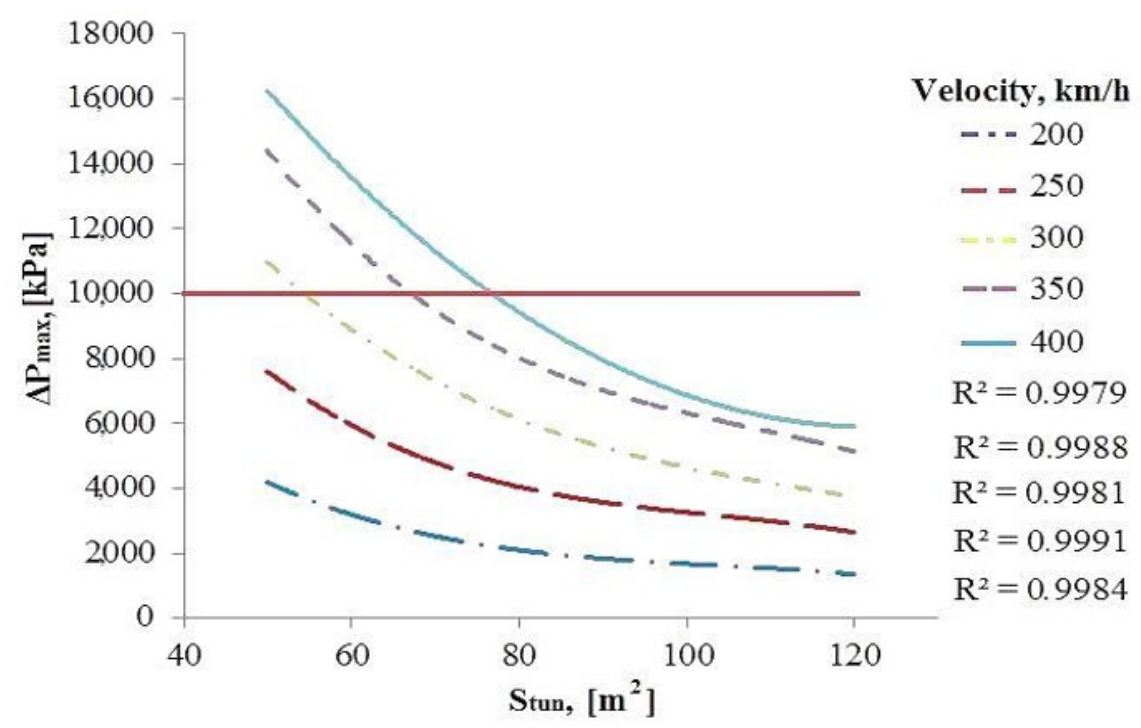

Fig. 6. Dependence of the aerodynamic pressure in the tunnel of the tunnel cross-sectional area and the train velocity (the horizontal line indicates the maximum safe pressure drop $\Delta \mathrm{P} \leq 10 \mathrm{kPa}$ )

The comparison criteria are the safety requirements in UIC 779-11, for which, as shown in the paper, the safety criterion for a pressure drop of $10 \mathrm{kPa}$ specified in the technical conditions of the Russian Federation (technical specifications developed including the PSTU (2014)). It may not be violated for the entire range of train velocities under study, but it does affect the optimized parameter of the tunnel cross-section area that is determined. For comparison of study results with normative values is an additional indicator - the blockage ratio $\beta$ equals to the division of the cross sectional area of train $S_{\text {tr }}$ (equal to the study $14.5 \mathrm{~m}^{2}$ ) to the cross sectional area of the tunnel $\mathrm{S}_{\mathrm{tun}}$.

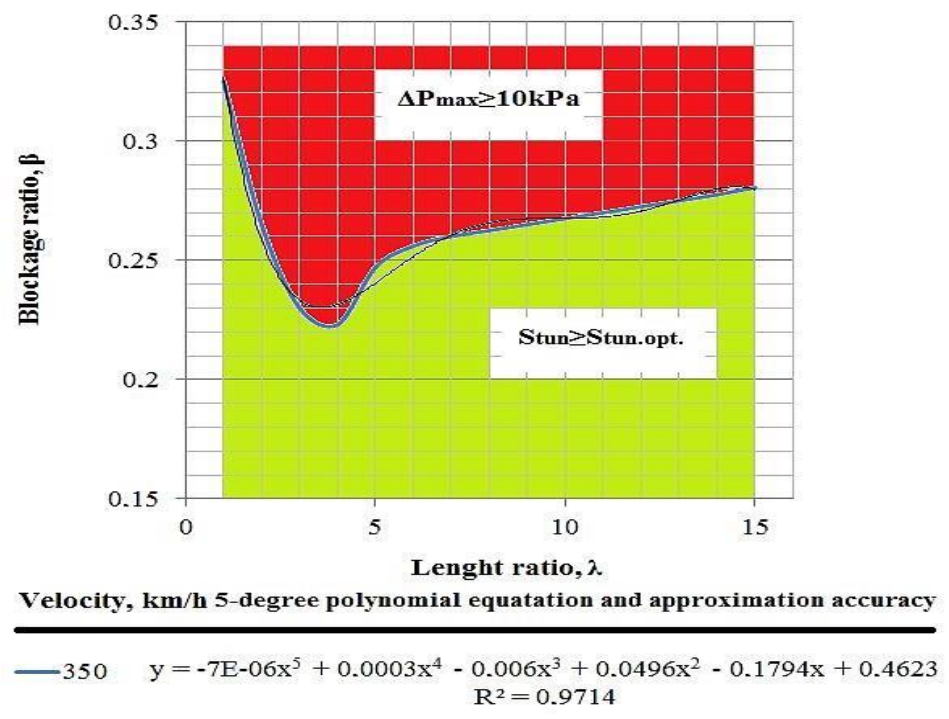

Fig.7. Diagram of the required blockage ratio dependence (to determine the optimal tunnel crosssectional area) on the train velocity in the tunnel $(350 \mathrm{~km} / \mathrm{h})$ and the length ratio in compliance with safety criterion for the pressure drop $\Delta \mathrm{P} \leq 10 \mathrm{kPa}$ 
This indicator allows one action unification the diversity of the world's rolling stock in solving tasks of aerodynamic impact: using the ratio of values, rather than a specific characteristic of an individual object (tunnel or train), it is possible to apply the safety criterion to the search for the optimal value of the cross-section area of the tunnel. Together with the use of the length ratio, which unifies the length of high-speed trains and tunnels, the graphical result of solving the task is easy to understand and easy to use.

Figure 7 is a diagram where the approximated curve shows the boundary of the critical pressure drop of $10 \mathrm{kPa}$. The optimum zone is theoretically located at this boundary. This means that in the lower zone of the graph there is an area of permissible values of the crosssection area of the tunnel that satisfy the optimization criterion for the pressure drop, and in the upper zone there are abundance of values that do not meet this condition - with such values of the blockage ratio $\beta$, the pressure drop at the specified train velocities will exceed the critical one. It is noted that the maximum pressure drop in the tunnel is also determined by the length ratio $\lambda$ and differs significantly at different values of $\lambda$, some of them determine the worst situations in the tunnel, thereby affecting the value $\beta$. This is due to the reflection of tunnel pressure waves that propagate over the entire length of the tunnel during the movement of the train, causing fluctuations and pressure drops that affect the possible excess of the critical pressure. Prediction of aerodynamic interaction in a multifactor system "tunnel-train" in high-speed train traffic determines the ability of pressure control through a comprehensive review of influencing factors and rationalization of the geometric parameters of the tunnel with a constant-velocity mode of movement of trains: knowing the cross sectional area of the tunnel built or designed, it becomes possible to determine the arising pressure, to minimize its.

The practical application of the graph in figure 7 is as follows: when designing a tunnel of a certain length, assigning a critical pressure amplitude and the velocity of train operation, it is possible to determine the required $S_{\text {tun }}$ area of tunnel cross section, which allows solving the most important of the considered tasks - determining the safe (optimal) cross-section area of the tunnel $S_{\text {tun.opt. }}$ depending on the velocity of the train. Thus, when a train is moving in a tunnel at $\mathrm{v}_{\mathrm{tr}}=350 \mathrm{~km} / \mathrm{h}$ with a length ratio that determines the worst conditions for pressure drops, in this case $\lambda=4$, in order to satisfy the safety criterion for a pressure drop $\Delta \mathrm{P} \leq 10 \mathrm{kPa}$, it is necessary to determine the boundary value of the blockageratio equal to $\beta=0.223$. In this study a model of a high-speed train with $\mathrm{S}_{\mathrm{tr}}=14.5 \mathrm{~m}^{2}$ is used, which allows to determine the optimal operating value of the cross-sectional area of

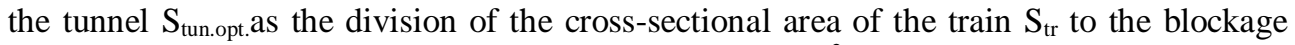
ratio $\beta$ : thus, the value $S_{\text {tun.opt. }}$ will be approximately $65.0 \mathrm{~m}^{2}$.

From the point of view of recommendations for the regulation of technical geometric parameters of a tunnel, such dependencies can be of a significant practical nature, which determines the prospects for further research.

\section{Conclusion}

The study established the applicability of the safety criterion $\Delta \mathrm{P} \leq 10 \mathrm{kPa}$ to the justification of the geometric parameters of tunnel structures on the HSR, and determined the aerodynamic parameters and loads in the set on the basis of the optimal experiment planning program. Its graphical interpretation in the form of dependencies between geometric and mechanical parameters of the multi-factor system "tunnel-train" in highspeed traffic allowed synthesizing the phenomena of aerodynamic interaction and presenting the result of predicting the behavior of the system in a visual form. The greatest probability of critical pressure occurs at high speeds of trains $(300-400 \mathrm{~km} / \mathrm{h})$ with a tunnel cross-section area of less than $77 \mathrm{~m}^{2}$. At the same time, the studied effect of the length of the structure on the amount of aerodynamic pressure showed that regardless of the tunnel 
cross-section area size, the greatest pressures are in the range of the length ratio from 2 to 6 , which should be taken into account when assigning the length of the operating on HSR rolling stock

This paper provides the following conclusions:

1) The accuracy and efficiency of the applied mathematical models are shown;

2) The reliability of the hypothesis and the results of calculations are proved;

3) Graphical dependencies of the "tunnel-train" system parameters are created;

4) The optimal values of the tunnel cross-sectional area on the HSR under the specified conditions are determined.

Solving the optimization task will be a step towards assigning practical recommendations for appointing technical characteristics of the system and the tunnel structure, and experimental confirmation and verification of the results will allow successfully setting technical parameters using the multi-factor system "tunnel-train". Solving these tasks makes it possible to increase the efficiency of high-speed rail transport. At the same time, the cost of construction investments and the current maintenance of the infrastructure object can be reduced by up to $25 \%$ with the specified technical characteristics.

\section{References}

1. F. Liu, S.Yao, J. Zhang, Y. Wang, Tunnelling and Underground Space Technology 72, 97-106 (2018). DOI:10.1016/j.tust.2017.11.018.

2. P. Derkowski, S. Clark., R. Strut, et.al., High-Speed Rail Aerodynamic Assessment and Mitigation Report (Final report, US, 2015)

3. P. Ricco, A. Baron, P. Molteni, Journal of Wind Engineering and Industrial Aerodynamics 95, 781-808 (2007). DOI:10.1016/j.jweia.2007.01.008

4. Yang, Mingzhi \& Du, Juntao \& Li, Zhiwei \& Huang, Sha \& Zhou, Dan. PLOS ONE 12, e0169471 (2017). DOI:10.1371/journal.pone.0169471.

5. T.Gilbert, C. Baker, A. Quinn, Journal of Rail and Rapid Transit 227, 609-622 (2013). DOI: 10.1177/0954409713494947

6. Weichao Yang, E. Deng, Mingfeng Lei, Pingping Zhang, Rongshen Yin, Journal of Wind Engineering and Industrial Aerodynamics 175, 229-243 (2018). DOI:10.1016/j.jweia.2018.01.018.

7. TCP EN 1991-2-2009 (02250) EUROCOD 2.Design of concrete structures. Traffic loads on bridges.

8. UIC code 779-11R. Determination of railway tunnel cross-sectional areas on the basis of aerodynamic considerations (2005)

9. Wang, Xiao, Journal of Vibroengineering 20, 1144-1160 (2018). DOI:10.21595/jve.2017.18430.

10. A.P. Ledyaev, V.N. Kavkazskiy, R.O. Kreer, Transport Urala 4(47), 3-9 (2015). DOI:10.20291/1815-9400-2015-4-3-9

11. Zhang Lei, Thurow Kerstin, Stoll Norbert, Liu Hui, Journal of Wind Engineering and Industrial Aerodynamics 178, 17 (2018). DOI:10.1016/j.jweia.2018.05.003

12. D. Heine, K. Ehrenfried, H. Kühnelt, S. Lachinger, M. Rudolph, A. Vorwagner, F. Saliger, Journal of Wind Engineering and Industrial Aerodynamics 189, 258-265 (2019). DOI: 10.1016/j.jweia.2019.03.0323 